\title{
The psycho-emotional impact of COVID-19 on surgical staff working in emergency departments
}

\author{
Vasileios Karampelias ${ }^{1} \cdot$ Dimitris Karonis $^{1} \cdot$ Varvara Psaroudi $^{1}$
}

Received: 5 May 2020 / Accepted: 28 May 2020 / Published online: 3 June 2020

c) Springer-Verlag GmbH Germany, part of Springer Nature 2020

COVID-19 is an infectious disease that attacks the respiratory system [1] first reported in the Chinese city of Wuhan in December 2019. It has since spread globally, resulting in a full-scale pandemic. On January 30th, 2020, the World Health Organization classified the outbreak as a Public Health Emergency of International Concern. As a result, governments around the globe have implemented drastic measures in an attempt to limit the burden on local healthcare systems. Nevertheless, the global number of patients continues to rise, with the USA, and Brazil having to cope with the largest COVID-19 prevalence [2].

Facing this disease is challenging; there is no known cure, its severity can rapidly fluctuate with no warning, and it can lead to multiple severe debilitating symptoms, such as dyspnea, fever, dizziness, cough, fatigue, and ultimately death [3]. Physicians and nurses comprise the frontline defense against COVID-19, which often involves surgery [4]. Therefore, the surgical staff working in emergency departments (EDs) are presently facing unprecedented challenges. They are used to working in an adverse, high-pressure environment, as according to Jensen, a typical ED treats 18,000 visits per year, which amounts to $8760 \mathrm{~h}$ of treatment [5]. Even so, the current global pandemic of COVID-19 has substantially inflated these numbers, as according to the WHO, the USA identified 12,000 confirmed patients with COVID19 between March and April, 2020 [6]. This indicates the rapid increase in emergency cases and commensurate effort required by ED surgical staff. The burden placed upon them demands these healthcare workers operate in peak condition both on a physical and a psychological level. However, a combination of increasing patient numbers and staff shortages threatens ED workers' psychological health as a result of extended working hours, fatigue, and burnout.

Varvara Psaroudi

psaroudi.v@gmail.com

1 Department of Surgery, School of Medicine, University of Patras, Patras, Greece
The psycho-emotional impact of the pandemic on surgical staff involves numerous factors that could potentially compromise mental wellbeing, affecting several aspects of our emotional profile, behavioral traits, and overall personality. Stress levels, levels of responses to fear, depression, and related coping mechanisms, feelings of helplessness, and how they are shaped, workload-related burnout, and anxiety are matters of concern in this context. Accordingly, it is reasonable to assume that the surgical staff working in EDs must also cope with a deteriorating psycho-emotional status. According to a study by Lai et al., "a considerable proportion of healthcare workers reported experiencing symptoms of depression, anxiety, insomnia, and distress" [7].

Taking a closer look at the above, we can understand how these factors influence the EDs' surgical staff performance. In particular, work-related stress has been observed to impact performance across numerous professions plagued by its root causes, i.e., excessive workload and lack of social support, as described by the American Psychological Association [8]. These also apply to the surgical staff working in EDs.

Fear is another factor that may influence the performance of ED surgical staff. Fear is not always a negative emotion, as it can ward against impulsive decisions that could lead to undesirable outcomes. However, the element of unfamiliarity in dealing with COVID-19 serves to enhance the levels of fear experienced by medical professionals, as our limited knowledge of the disease instinctively generates an additional layer of suspicion, a disposition exacerbated by the lack of basic protective equipment for the physicians who need it the most. ED surgical staff are also faced with a fear of potential COVID-19 infection. This is understandable; they are working in the front lines and are exposed to numerous patients on a daily basis. Moreover, the conditions under which they are working are not ideal, according to the following testimony by Dr. Samantha Batt-Rawden, the president of the UK Doctors' Association (DAUK): 'Lack of personal protective equipment continues to be a critical 
issue. It is heartbreaking to hear that some staff have been told to simply 'hold their breath' due to lack of masks" [9]. According to another DAUK source, "it has become increasingly clear that there is a dire shortage of appropriate personal protective equipment for health and social care workers" [10]. The resulting levels of fear can severely impact individual performance and lead to a higher probability of work-related errors. Individual response to fear is personality dependent and thus cannot be predicted or measured. Consequently, it becomes vital that surgical staff are constantly trained and remain up to date with emerging coping strategies throughout their careers.

Depression is a mood disorder affecting the overall mental state, making no exceptions in terms of its prevalence. It affects people across ethnicities, religions, professions, and economic backgrounds. Depression is an overwhelming state of sadness that hinders the ability to perform everyday tasks, including work. The overall state of depression involves feelings of helplessness; this can prove a serious factor affecting the ED surgical staff's state of mind when manifesting, inevitably influencing their decision-making process. Other common symptoms include sleep disturbances. According to a survey by Yan et al., the medical staff of Chinese hospitals who have treated patients with COVID-19 have reported experiencing depression and sleep disorders [11].

When faced with overwhelming numbers of patients, any medical worker, let alone ED surgical staff, can display such depressive symptoms. As the COVID-19 prevalence is alarmingly increasing with no cure in sight, the surgical staff working in EDs are threatened with depression. Coping strategies involve dietary, habitual, and exercise interventions. However, it becomes challenging to promote such lifestyle changes for individuals overburdened with demanding work conditions, as interventions require high levels of personal discipline, free time, and a clear mental state.

Burnout constitutes emotional exhaustion, which could be detrimental to workplace relationships and overall work performance. According to Wilson, an excessive workload is the primary cause of burnout [12]. Resting and interacting with family and loved ones are the best means through which burnout can be avoided. However, respite is unfeasible when hospitals remain understaffed, underequipped, and unprepared to face a pandemic.

Having a deteriorating mental state leads to errors; ED surgical staff cannot afford to make any. Therefore, their mental state must be monitored, supported, and treated whenever necessary. Nevertheless, this is a practically impossible task during such a pressing period, when physical health issues are foregrounded and prioritized. According to Chen et al.: "maintaining staff mental health is essential to better control infectious diseases" [13], indicating the necessity of maintaining a good status of mental health for ED surgical staff. Hospitals must be properly and adequately staffed to allow medical workers to dedicate themselves to the fight against COVID-19.

Other factors that can contribute to maintain a healthy workplace is the establishment of a workplace-related culture that promotes balanced and substantial rewards. According to the WHO: "An imbalance between effort and reward may result in a sense of injustice or unfairness in workers, leading to feelings of anger that may be directed against a supervisor or co-worker" [14].

In addition, psychotherapy sessions must be introduced to ED surgical staff to ensure that their mental state will not impair their performance. According to Folkman and Greer, it is important to encourage clinicians to be attentive to their psychological health, the same way they are towards their physical well being [15].

Concluding, the psycho-emotional impact of the COVID19 pandemic on ED surgical staff is indeed multifaceted; it influences both their ability to work and their ability to cope with mental health issues. In addition, the situation is only worsened by basic protective gear shortages, excessive working hours, and overwhelming patient numbers. Ensuring both the staff's physical and mental health is crucial for maintaining a high level of healthcare provision. All governments must invest in human capital, protective gear, and proper mental health improvement strategies to ensure the best possible conditions in EDs.

\section{Availability of data and material}

Not applicable.

Acknowledgements We would like to thank "The Sciencing Team" (https://thesciencingteam.com) for the thorough English editing of our manuscript and for publication support.

Funding None.

Conflict of interest The authors declare that they have no competing interests.

Ethics approval Not applicable.

Consent to participate Not applicable.

Consent for publication Not applicable.

Code availability Not applicable.

\section{References}

1. Coronavirus: what happens when you get infected. WebMD. https ://www.webmd.com/lung/coronavirus-covid-19-affects-body\#1. Accessed 24 Apr 2020. 
2. World Health Organization. Coronavirus disease (COVID-19) situation report-132. https://www.who.int/docs/default-source/ coronaviruse/situation-reports/20200531-covid-19-sitrep-132. pdf?sfvrsn=d9c2eaef_2. Accessed 1 June 2020.

3. World Health Organization. Q\&A on coronaviruses (COVID-19). 2020. https://www.who.int/news-room/q-a-detail/q-a-coronaviru ses. Assessed 24 Apr 2020.

4. American College of Surgeons. COVID-19: guidance for triage of non-emergent surgical procedures. 2020. https://www.facs.org/ covid-19/clinical-guidance/triage. Accessed 24 April 2020.

5. Jensen KB. Staffing your emergency department. Best practices. 2020. https://www.best-practices.com/wp-content/uploa ds/2015/08/Staffing-Your-ED_White-Paper.pdf. Accessed 24 April 2020.

6. Roser M, Ritchie H. Coronavirus pandemic (COVID-19)-the data. Our World in data. https://ourworldindata.org/coronaviru s-data. Accessed 24 April 2020.

7. Lai J, Ma S, Wang Y, et al. Factors associated with mental health outcomes among health care workers exposed to COVID-19. JAMA Netw Open. 2020;3(3):e203976. https://doi.org/10.1001/ jamanetworkopen.2020.3976.

8. American Psychological Association. Coping with stress at work. American Psychological Association. https://www.apa.org/helpc enter/work-stress. Accessed 24 April 2020.

9. Campell D. Doctors lacking PPE 'bullied' into treating Covid-19 patients. The Guardian. 2020. https://www.theguardian.com/world /2020/apr/06/nhs-doctors-lacking-ppe-bullied-into-treating-covid -19-patients. Accessed 24 April 2020.
10. Applebee J, Parmar R, Marlowe G, Forster M, O'Sullivan T. DAUK co-signs joint statement from doctors' unions: the lack of safe PPE will be this government's legacy. Dauk. 2020. https:// www.dauk.org/news/2020/4/6/jointstatementdoctorsinuniteppe. Accessed 24 Apr 2020.

11. Lv Y, Zhang Z, Zeng W, Li J, Wang X, Luo GH. Anxiety and depression survey of Chinese medical staff before and during COVID-19 defense. SSRN Electron J. 2020. https://doi. org/10.2139/ssrn.3551350.

12. Wilson SM. Avoid the burn. American Psychological Association. 2011. https://www.apa.org/gradpsych/2011/03/corner. Accessed 24 April 2020.

13. Chen Q, Liang M, Li Y, et al. Mental health care for medical staff in China during the COVID-19 outbreak. Lancet Psychiatry. 2020;7(4):e15-6. https://doi.org/10.1016/s2215-0366(20)30078 $-\mathrm{x}$.

14. Burton J. Healthy workplace framework and model: background and supporting literature and practice. World Health Organization. 2010. https://www.who.int/occupational_health/healthy_workp lace_framework.pdf. Accessed 24 April 2020.

15. Folkman S, Greer S. Promoting psychological well-being in the face of serious illness: when theory, research and practice inform each other. Psychooncology. 2020;9(1):11-9. https:// doi.org/10.1002/(sici)1099-1611(200001/02)9:1<11:aid-pon42 4>3.0.co;2-z. 